PROCEEDINGS OF THE

AMERICAN MATHEMATICAL SOCIETY

Volume 139, Number 4, April 2011, Pages 1421-1432

S 0002-9939(2010)10612-3

Article electronically published on September 2, 2010

\title{
ASYMPTOTIC BEHAVIOR \\ FOR A NONLOCAL DIFFUSION EQUATION \\ WITH ABSORPTION AND NONINTEGRABLE INITIAL DATA. THE SUPERCRITICAL CASE
}

\author{
JOANA TERRA AND NOEMI WOLANSKI
}

(Communicated by Matthew J. Gursky)

\begin{abstract}
In this paper we study the asymptotic behavior as time goes to infinity of the solution to a nonlocal diffusion equation with absorption modeled by a powerlike reaction $-u^{p}, p>1$ and set in $\mathbb{R}^{N}$. We consider a bounded, nonnegative initial datum $u_{0}$ that behaves like a negative power at infinity. That is, $|x|^{\alpha} u_{0}(x) \rightarrow A>0$ as $|x| \rightarrow \infty$ with $0<\alpha \leq N$. We prove that, in the supercritical case $p>1+2 / \alpha$, the solution behaves asymptotically as that of the heat equation (with diffusivity $\mathfrak{a}$ related to the nonlocal operator) with the same initial datum.
\end{abstract}

\section{INTRODUCTION}

In this paper we study the asymptotic behavior as time goes to infinity of the solution to a nonlocal diffusion equation in $R^{N}$ with an absorption term. We are interested in the case in which the initial datum is nonintegrable. More precisely, we consider a bounded datum $u_{0}(x)$ such that $|x|^{\alpha} u_{0}(x) \rightarrow A>0$ as $|x| \rightarrow \infty$ with $0<\alpha \leq N$. The problem under consideration is the following:

$$
\begin{aligned}
& u_{t}(x, t)=\int J(x-y)(u(y, t)-u(x, t)) d y-u^{p}(x, t), \\
& u(x, 0)=u_{0}(x),
\end{aligned}
$$

where $J \in C_{0}^{\infty}\left(\mathbb{R}^{N}\right)$, radially symmetric with $J \geq 0$ and $\int J=1$.

The term $\int J(x-y)(u(y, t)-u(x, t)) d y$ in (1.1) represents diffusion. In fact, let $u(x, t)$ be the density of a population at the point $x$ at time $t$. The kernel $J(x-y)$ may be seen as the probability distribution density of jumping from site $y$ to site $x$. Thus, $\int J(x-y) u(y, t) d y$ represents the rate at which the population is arriving at the site $x$ from all over space. By the symmetry of the kernel $J, \int J(x-y) u(x, t) d y$ then represents the rate at which it is leaving the point $x$.

The absorption term $-u^{p}(x, t)$ in the equation represents a rate of consumption due to an internal reaction.

This diffusion operator has been used to model several nonlocal diffusion processes in the last few years. See for instance [1, 2, 3, 4, 9, 19. In particular,

Received by the editors November 25, 2009 and, in revised form, April 29, 2010.

2010 Mathematics Subject Classification. Primary 35K57, 35B40.

Key words and phrases. Nonlocal diffusion, boundary value problems.

(C)2010 American Mathematical Society 
nonlocal diffusions are of interest in biological and biomedical problems. Recently, these kinds of nonlocal operators have also been used for image enhancement [10.

It can be seen that, when properly rescaled, the nonlocal operator approximates $\mathfrak{a} \Delta$, where $\Delta$ is the Laplace operator and $\mathfrak{a}$ is a constant that depends only on $J$ and the dimension $N$. (See, for instance, 7,8 .)

Another close relation to the heat operator with diffusivity $\mathfrak{a}$ was found in [5, 13, where it was proven that for bounded and integrable initial data, the asymptotic behavior as $t$ tends to infinity of the solution $u_{L}$ to the equation without absorption,

$$
u_{t}(x, t)=\int J(x-y)(u(y, t)-u(x, t)) d y=L u,
$$

is the same as that of the solution of the heat equation with diffusivity $\mathfrak{a}$ and the same initial condition. Namely,

$$
t^{N / 2}\left\|u(x, t)-M U_{\mathfrak{a}}(x, t)\right\|_{L^{\infty}(|x| \leq K \sqrt{t})} \rightarrow 0 \quad \text { as } t \rightarrow \infty \quad \forall K>0,
$$

with $U_{\mathfrak{a}}$ the fundamental solution of the heat equation with diffusivity $\mathfrak{a}$ and $M=$ $\int u_{0}$ the initial mass. The assumption on the initial condition is that $u_{0} \in L^{\infty} \cap L^{1}$.

In a recent paper [17, the nonlocal equation with absorption (1.1) was considered in the case $u(x, 0) \in L^{\infty} \cap L^{1}$ and $p>1+2 / N$. The authors prove that $u$ also satisfies (1.3). Some results for $p<1+2 / N$ can also be found in that paper. The critical case $p=1+2 / N$ was left open.

On the other hand, the asymptotic behavior of the solutions to the heat equation and of the heat equation with absorption are very well known for some nonintegrable initial data with a precise power-like behavior at infinity. In fact, Kamin and Peletier in [14] study the asymptotic behavior of the solution to

$$
\begin{array}{ll}
u_{t}=\mathfrak{a} \Delta u-u^{p} & \text { in } \mathbb{R}^{N} \times(0, \infty), \\
u(x, 0)=u_{0}(x) & \text { in } \mathbb{R}^{N},
\end{array}
$$

with $u_{0} \in L^{\infty}$ and $u_{0} \geq 0$ such that

$$
|x|^{\alpha} u_{0}(x) \rightarrow A>0 \quad \text { as }|x| \rightarrow \infty,
$$

with $0<\alpha<N$. In fact, the authors prove that

$$
t^{\alpha / 2}\left\|u(x, t)-U_{\alpha, A}(x, t)\right\|_{L^{\infty}(|x| \leq K \sqrt{t})} \rightarrow 0 \quad \text { as } t \rightarrow \infty \quad \forall K>0,
$$

where $U_{\alpha, A}$ is the solution to

$$
\begin{aligned}
& U_{t}=\mathfrak{a} \Delta U-\delta_{p, \alpha} U^{p} \quad \text { in } \mathbb{R}^{N} \times(0, \infty), \\
& U(x, 0)=\frac{A}{|x|^{\alpha}} \quad \text { in } \mathbb{R}^{N} .
\end{aligned}
$$

Here $\delta_{p, \alpha}=0$ in the supercritical case $p>1+2 / \alpha$ and $\delta_{p, \alpha}=1$ in the critical case $p=1+2 / \alpha$.

Then, in [15, Kamin and Ughi study the case where $\alpha=N$ in (1.5) and $p>$ $1+2 / N$. They prove that

$$
t^{N / 2}\left\|\frac{u(x, t)}{\log t}-C_{A, N} U_{\mathfrak{a}}(x, t)\right\|_{L^{\infty}(|x| \leq K \sqrt{t})} \rightarrow 0 \quad \text { as } t \rightarrow \infty \quad \forall K>0,
$$

with $C_{A, N}$ a constant that depends only on $A$ and $N$, and $U_{\mathfrak{a}}$ is the fundamental solution of the heat operator with diffusivity $\mathfrak{a}$. 
Later on, in 12, Herraiz fully analyzes problem (1.4) and presents a complete characterization including the asymptotic behavior outside the parabolas $|x| \leq$ $K \sqrt{t}$. Also in [12, Herraiz gives detailed proofs of the previous results on the asymptotic behavior of solutions $u_{\Delta}$ of the heat equation without absorption with initial data satisfying (1.5) for every $\alpha$. In particular, he finds that

$$
\left\|u_{\Delta}\right\|_{L^{\infty}\left(\mathbb{R}^{N}\right)} \leq\left\{\begin{aligned}
C t^{-\alpha / 2}, & \text { for } \quad \alpha<N \\
C t^{-N / 2} \log t, & \text { for } \quad \alpha=N .
\end{aligned}\right.
$$

The case where $\alpha<N$ is also considered in 14 and the case $\alpha=N$ in also analysed in 15, as a particular case of the porous medium equation. In fact, note that the results of [14, 15] apply also to the solution $u_{\Delta}$ of the homogeneous heat equation. In this case, the constant $\delta_{\alpha, A}$ in (1.7) is zero so that $U_{\alpha, A}$ is a solution to the homogeneous heat equation.

It is the purpose of this paper to prove that for initial data satisfying (1.5) with $0<\alpha \leq N$ and $p>1+2 / \alpha$, the asymptotic behavior of the solution to the nonlocal diffusion problem with absorption (1.1) is the same as the asymptotic behavior of solutions to the heat equation with absorption (1.4), that is, that (1.6) holds in the case $\alpha<N$ and (1.8) holds in the case $\alpha=N$ with $u$ the solution of (1.1).

The proofs of these results rely strongly on estimates for the fundamental solution of the equation without absorption (1.2) .

In [5] the authors observed that the fundamental solution of the operator in (1.2) can be written as

$$
U(x, t)=e^{-t} \delta+W(x, t),
$$

where $\delta$ is the Dirac measure and $W(x, t)$ is a smooth function.

Then, in $\left[13\right.$ the authors established $L^{\infty}$ estimates for $W$ and proved that

$$
t^{N / 2}\left\|W(x, t)-U_{\mathfrak{a}}(x, t)\right\|_{L^{\infty}\left(\mathbb{R}^{N}\right)} \rightarrow 0 \quad \text { as } t \rightarrow \infty,
$$

where $U_{\mathfrak{a}}$ is the fundamental solution of the heat equation with diffusivity $\mathfrak{a}$.

This estimate allowed them to prove that the solution of the homogeneous nonlocal diffusion equation (1.2) with bounded and integrable initial data behaves, for $t$ tending to infinity, as the solution of the heat equation with diffusivity $\mathfrak{a}$, removing in this way the hypothesis that the Fourier transform of $u_{0}$ be in $L^{1}$ as was assumed in 5 .

In the present paper, as we are considering nonintegrable initial data, these $L^{\infty}$ estimates cannot be used. So, the first step is to get estimates in $L^{q}$-norms for $1 \leq q<\infty$.

As in [17, the philosophy of the study of the asymptotic behavior for the problem with absorption in the supercritical case is that, as time tends to infinity, the absorption term goes to 0 so fast that the behavior is the same as that of the equation without absorption. Thus, what we need to prove is essentially that, as time tends to infinity, the difference between the solutions to both problems goes to 0 faster than each term separately.

In order to prove this result it is necessary to compare the solution $u$ of (1.1) with the solution $u_{L}$ of the equation without absorption (1.2) that coincides with $u$ at a large time $t_{0}$. Once we prove that this difference is small the result follows if we know that the asymptotic behavior of $u_{L}$ is the same as that of (1.4). To this end, we first analyze the asymptotic behavior for the nonlocal equation without absorption (1.2) with an initial datum satisfying (1.5). In order to finish the argument, it is 
essential to know, and we prove it in this paper, that the behavior in space of the solution of our problem (1.1) at any time $t_{0}$ is the same as that of the initial datum $u_{0}$, that is, that $u\left(\cdot, t_{0}\right)$ satisfies (1.5) at any positive time $t_{0}$.

The techniques of this paper do not work in the critical case $p=1+2 / \alpha$. In fact, for such a $p$, the asymptotic behavior is that of the heat equation with absorption since the diffusion and the absorption terms are of the same order. We have to proceed in a completely different way by going back to the techniques of the original paper for the heat equation [14, and we do so in [18. The method in [18. also works in the supercritical case considered in this paper. Nevertheless, the ideas in the present paper are simpler and rather easy to implement, and therefore we consider that it is worth presenting them separately.

The paper is organized as follows. In Section 2 we prove the asymptotic estimates of $W$ in $L^{q}$-norms for finite $q$ and use them to study the behavior of the solution $u_{L}$ of the linear equation (1.2) with a nonnegative, bounded initial datum satisfying (1.5) with $0<\alpha \leq N$.

In particular, we prove that the solution $u_{L}$ satisfies

$$
t^{\alpha / 2}\left\|u_{L}(x, t)-u_{\Delta}(x, t)\right\|_{L^{\infty}\left(\mathbb{R}^{N}\right)} \rightarrow 0 \quad \text { as } t \rightarrow \infty,
$$

where $u_{\Delta}$ is the solution of the heat equation with diffusivity $\mathfrak{a}$ and initial condition $u_{L}(x, 0)$.

Moreover, in this section we establish the precise behavior of $u_{L}$ for $|x| \rightarrow \infty$.

In Section 3 we prove the global existence and uniqueness of the solution to (1.1), with a more general absorption term $-|u|^{p-1} u$, for our nonintegrable initial data with power-like behavior at infinity. We also establish that the solution is bounded, in any time interval $[0, T]$, by $C_{T}(1+|x|)^{-\alpha}$ for a certain constant $C_{T}$. Then, when $u_{0}$ is nonnegative, we deduce that the solution is nonnegative. Finally, we prove that for every $t_{0}>0$,

$$
|x|^{\alpha} u\left(x, t_{0}\right) \rightarrow A \quad \text { as }|x| \rightarrow \infty .
$$

Finally, in Section 4 we prove our main result. That is, we prove that (1.6) holds when $\alpha<N$ and (1.8) when $\alpha=N$ with $u$ the solution to (1.1).

\section{Preliminary Results And homogeneous equation}

In this section we study the asymptotic behavior of bounded solutions to the homogeneous equation

$$
\begin{aligned}
& u_{t}=\int J(x-y)(u(y, t)-u(x, t)) d y \quad \text { in } \mathbb{R}^{N} \times(0, \infty), \\
& u(x, 0)=u_{0}(x) \quad \text { in } \mathbb{R}^{N} .
\end{aligned}
$$

Existence of bounded solutions for bounded initial data is a well known result that can be seen by different arguments. One possible construction is to convolve the initial datum with the fundamental solution (1.10). The uniqueness of bounded solutions follows from the comparison principle for bounded solutions. (See [16], Proposition 2.2 with $\theta=0$ for the case of smooth solutions. In order to get the result in our case we just have to approximate the initial datum by smooth functions that are uniformly bounded.)

In this section we first obtain estimates in $L^{q}\left(\mathbb{R}^{N}\right)$ for the "good" part $W(x, t)$ of the fundamental solution of the nonlocal diffusion equation. 
Then, we get the result on the asymptotic behavior as time goes to infinity of the solution of the equation for initial data that behave as a negative power at infinity.

Finally, we prove that the solution behaves as the initial datum for every $t>0$.

Let us recall (see [5]) that the fundamental solution of the equation (1.2) is given by

$$
U(x, t)=e^{-t} \delta+W(x, t),
$$

where $W$ is a smooth function. In fact, by taking the Fourier transform in the space variables, the authors show that $W(\cdot, t)$ belongs to the Schwartz class of $C^{\infty}$ functions rapidly decaying at infinity together with their derivatives.

Moreover, in [13], by using the characterization of $W$ in terms of its Fourier transform, the authors prove that

$$
t^{N / 2}\left\|W(x, t)-U_{\mathfrak{a}}(x, t)\right\|_{L^{\infty}\left(\mathbb{R}^{N}\right)} \leq C t^{-1 / 2},
$$

where $U_{\mathfrak{a}}$ is the fundamental solution of the heat equation with diffusivity $\mathfrak{a}$.

The Fourier transform method allows us to get $L^{q}$ estimates only for $q \geq 2$. In order to get an $L^{1}$ estimate we observe that $W$ is the solution to

$$
\begin{aligned}
& W_{t}(x, t)=\int J(x-y)(W(y, t)-W(x, t)) d y+e^{-t} J(x), \\
& W(x, 0)=0 .
\end{aligned}
$$

In fact, let us call $L$ the nonlocal operator. This is $L u=J * u-u$. Then,

$$
\begin{aligned}
W_{t}(x, t)-L W(x, t) & =U_{t}(x, t)-L U(x, t)-\frac{\partial}{\partial t} e^{-t} \delta+e^{-t} L \delta(x) \\
& =e^{-t} \delta+e^{-t}(J * \delta-\delta)(x) \\
& =e^{-t} J(x) .
\end{aligned}
$$

Moreover, since $U(x, 0)=\delta$, it follows that $W(x, 0)=0$.

In particular, by the comparison principle, $W \geq 0$. On the other hand, since the unique bounded solution to the homogeneous nonlocal diffusion equation with initial datum $u_{0} \equiv 1$ is the function $u(x, t) \equiv 1$, the representation of this solution gives

$$
1=u(x, t)=e^{-t} u_{0}(x)+\int W(x-y, t) u_{0}(y) d y=e^{-t}+\int W(y, t) d y .
$$

Thus, $\int W(y, t) d y=1-e^{-t} \leq 1$ for every $t$.

Now, since $\int U_{\mathfrak{a}}(y, t) d y=1$ for every $t$, interpolation with inequality (2.3) gives the estimate

$$
\left\|W(x, t)-U_{\mathfrak{a}}(x, t)\right\|_{L^{q^{\prime}}\left(\mathbb{R}^{N}\right)} \leq C_{q} t^{-\frac{N+1}{2 q}} \quad \text { if } \quad \frac{1}{q}+\frac{1}{q^{\prime}}=1 .
$$

In particular, for every $1 \leq q \leq \infty$,

$$
\|W(x, t)\|_{L^{q^{\prime}}\left(\mathbb{R}^{N}\right)} \leq C_{q} t^{-\frac{N}{2 q}}
$$

With this estimate we can prove that for large $t$ the solution $u$ to (2.1) behaves as the solution of the heat equation with diffusivity a. In fact,

Theorem 2.1. Let $u_{0} \in L^{\infty}$ be nonnegative and such that there exist $0<\alpha \leq N$ and $A>0$ with $|x|^{\alpha} u_{0}(x) \rightarrow A$ as $|x| \rightarrow \infty$. Let $u$ be the solution of the equation 
$u_{t}=L u$ with initial condition $u_{0}$. Then, for every $0<\mu<\frac{\alpha}{2 N}$ there exists a constant $C_{\mu}$ such that

$$
t^{\alpha / 2}\left\|u(x, t)-u_{\Delta}(x, t)\right\|_{L^{\infty}\left(\mathbb{R}^{N}\right)} \leq C_{\mu} t^{-\mu} .
$$

In particular, if $\alpha<N$, due to the behavior of $u_{\Delta}$ (see (1.9) ) it follows that $u(x, t) \leq C t^{-\alpha / 2}$ and

$$
t^{\alpha / 2}\left\|u(x, t)-U_{\alpha, A}(x, t)\right\|_{L^{\infty}(|x| \leq K \sqrt{t})} \rightarrow 0 \quad \text { as } t \rightarrow \infty \quad \forall K>0,
$$

where $U_{\alpha, A}$ is the solution to (1.7).

If $\alpha=N$, from (1.9), it follows that $u(x, t) \leq C t^{-N / 2} \log t$ and

$$
t^{N / 2}\left\|\frac{u(x, t)}{\log t}-C_{A, N} U_{\mathfrak{a}}(x, t)\right\|_{L^{\infty}(|x| \leq K \sqrt{t})} \rightarrow 0 \quad \text { as } t \rightarrow \infty \quad \forall K>0,
$$

where $U_{\mathfrak{a}}$ is the fundamental solution of the heat operator with diffusivity $\mathfrak{a}$ and $C_{A, N}$ is a constant that depends only on $A$ and $N$.

Proof. Since $u_{0} \in L^{q}\left(\mathbb{R}^{N}\right)$ if $q \alpha>N$, by taking $q=\frac{N}{\alpha} \frac{1}{1-\varepsilon}, \varepsilon>0$, and applying (2.5) we get

$$
\begin{aligned}
& \left\|\int W(x-y, t) u_{0}(y) d y-\int U_{\mathfrak{a}}(x-y, t) u_{0}(y) d y\right\|_{L^{\infty}\left(\mathbb{R}^{N}\right)} \\
& \leq C_{q}\left\|u_{0}\right\|_{q} t^{-\frac{N+1}{2 q}}=C_{\varepsilon} t^{-\frac{\alpha}{2}(1-\varepsilon)-\frac{\alpha}{2 N}(1-\varepsilon)} \\
& =C_{\varepsilon} t^{-\frac{\alpha}{2}} t^{-\frac{\alpha}{2 N}+\varepsilon \frac{\alpha}{2}\left(1+\frac{1}{N}\right)}=C_{\mu} t^{-\frac{\alpha}{2}} t^{-\mu},
\end{aligned}
$$

with $0<\mu<\frac{\alpha}{2 N}$ arbitrary.

Thus,

$$
\left\|u(\cdot, t)-u_{\Delta}(\cdot, t)\right\|_{L^{\infty}\left(\mathbb{R}^{N}\right)} \leq e^{-t}\left\|u_{0}\right\|_{L^{\infty}\left(\mathbb{R}^{N}\right)}+C_{\mu} t^{-\frac{\alpha}{2}} t^{-\mu},
$$

and (2.7) holds.

The asymptotic behavior of $u$ follows immediately from (2.7) together with (1.6) and (1.8) respectively applied to the solution $u_{\Delta}$ of the homogeneous heat equation (see [14, 15]).

Finally, we prove that $u$ behaves at each positive time as its initial datum.

Proposition 2.1. Let $u$ be the solution of (2.1) with an initial datum $u_{0} \in L^{\infty}$ such that $|x|^{\alpha} u_{0}(x) \rightarrow A>0$ as $|x| \rightarrow \infty$ for some $\alpha>0$. Then,

(1) For every $T>0$ there exists a constant $C_{T}>0$ such that $|u(x, t)| \leq \frac{C_{T}}{(1+|x|)^{\alpha}}$ if $0 \leq t \leq T$.

(2) $|x|^{\alpha} u(x, t) \rightarrow A$ as $|x| \rightarrow \infty$ uniformly for $t$ bounded.

Proof. The proof follows from a fixed point argument. In fact, $u$ is a fixed point of the operator

$$
\mathcal{T} v(x, t)=e^{-t} u_{0}(x)+\int_{0}^{t} \int e^{-(t-s)} J(x-y) v(y, s) d y d s .
$$

Reciprocally, every bounded function that is a fixed point of the operator $\mathcal{T}$ in $\mathbb{R}^{N} \times\left(0, t_{0}\right)$ is a bounded solution of problem (2.1). By uniqueness, it coincides with $u$ in that time interval. 
Thus, in order to prove the proposition we will show that $\mathcal{T}$ has a fixed point in the set

$$
\begin{array}{r}
\mathcal{K}:=\left\{\left.v \in L^{\infty}\left(\mathbb{R}^{N} \times\left(0, t_{0}\right)\right)\left|(1+|x|)^{\alpha}\right| v(x, t)|\leq 2 B,| x\right|^{\alpha} v(x, t) \rightarrow A \text { as }|x| \rightarrow \infty\right. \\
\text { uniformly with respect to } \left.t \in\left[0, t_{0}\right]\right\} .
\end{array}
$$

The time step $t_{0}$ will be independent of the constant $B$, where $B$ is a bound of $(1+|x|)^{\alpha}\left|u_{0}(x)\right|$. Therefore, we can proceed inductively and find a constant for any time interval, thus proving (1). Moreover, (2) follows immediately from this argument.

So, let $v \in \mathcal{K}$ and let $w=\mathcal{T} v$. Then, since the support of $J$ is contained in a ball $B_{R}$ and $|x-y| \leq R$ implies that $|x| \leq R+|y|$, by assuming that $R \geq 1$ we get

$$
\begin{aligned}
(1 & +|x|)^{\alpha}|w(x, t)| \leq e^{-t}(1+|x|)^{\alpha}\left|u_{0}(x)\right| \\
& +\int_{0}^{t} \int e^{-(t-s)} J(x-y)(1+|x|)^{\alpha}|v(y, s)| d y d s \\
& \leq e^{-t} B+\int_{0}^{t} \int e^{-(t-s)} J(x-y)\left(1+(R+|y|)^{\alpha}\right)|v(y, s)| d y d s \\
& \leq B+(R+1)^{\alpha} 2 B t \leq 2 B
\end{aligned}
$$

if $t \leq t_{0}=\frac{1}{2}(R+1)^{-\alpha}$.

Now, let us prove that $|x|^{\alpha} w(x, t) \rightarrow A$ as $|x| \rightarrow \infty$ uniformly for $t \in\left(0, t_{0}\right)$. In fact, if $|x-y|<R$, it follows that $|y|>|x|-R$ and, if $|x| \geq|y|$,

$$
\left|\frac{|x|}{|y|}-1\right|<\frac{R}{|y|}<\frac{R}{|x|-R} .
$$

Now, if $|x|<|y|$,

$$
\left|\frac{|x|}{|y|}-1\right|=\frac{|y|-|x|}{|y|} \leq \frac{|x-y|}{|y|} \leq \frac{R}{|x|-R} .
$$

Thus, if $|x|$ is large enough, $\left|\frac{|x|^{\alpha}}{|y|^{\alpha}}-1\right| \leq \varepsilon$.

Therefore,

$$
\begin{aligned}
& \left.\left|\int J(x-y)\right| x\right|^{\alpha} v(y, s) d y-A \mid \\
& \quad \leq\left.\int J(x-y)|| y\right|^{\alpha} v(y, s)-\left.A|d y+| \int J(x-y)|| x\right|^{\alpha}-|y|^{\alpha}|| v(y, s)|d y| \\
& \quad \leq \int_{|y|>|x|-R} J(x-y) \varepsilon d y+\varepsilon \int J(x-y)|y|^{\alpha}|v(y, s)| d y<(1+2 B) \varepsilon
\end{aligned}
$$

if $|x|$ is large enough.

Finally,

$$
\begin{aligned}
\left.|| x\right|^{\alpha} w(x, t)-A \mid \leq & e^{-t}\left(|x|^{\alpha} u_{0}(x)-A\right) \\
& +\left.\int_{0}^{t} e^{-(t-s)}\left|\int J(x-y)\right| x\right|^{\alpha} v(y, s) d y-A \mid d s \\
< & 2(1+B) \varepsilon .
\end{aligned}
$$

This ends the proof. 


\section{Existence, Uniqueness And First PROPERTIES OF THE SOLUTion}

In this section we prove existence, uniqueness and time local properties of the solution to the nonlocal diffusion equation with absorption

$$
\begin{aligned}
& u_{t}=L u-|u|^{p-1} u, \\
& u(x, 0)=u_{0}(x) .
\end{aligned}
$$

In particular, we are interested in nonnegative bounded initial data that behave as a negative power at infinity, that is, which satisfy (1.5).

Local existence and uniqueness of the solution to 3.1) with $p>1$ and bounded initial datum follow by a fixed point argument. For instance, if we call $S(t)$ the semigroup associated to the equation $u_{t}=L u$ in $L^{\infty}\left(\mathbb{R}^{N}\right)$, we can find the solution as a fixed point in $\mathcal{K}:=\left\{v \in L^{\infty}\left(\mathbb{R}^{N} \times\left(0, t_{0}\right)\right) \mid\|v\|_{\infty} \leq 2\left\|u_{0}\right\|_{\infty}\right\}$ of the operator

$$
\mathcal{T} v=S(t) u_{0}-\int_{0}^{t} S(t-s)|v(x, s)|^{p-1} v(x, s) d s .
$$

In fact, it is easy to see that for small $t_{0}$ the operator $\mathcal{T}$ is a contraction from $\mathcal{K}$ to $\mathcal{K}$. Thus, there exists a bounded solution in the time interval $\left(0, t_{0}\right)$. Since positive constants are supersolutions and negative constants are subsolutions to problem (3.1), and the function $\tau \rightarrow|\tau|^{p-1} \tau$ is locally Lipschitz, the comparison principle (see, for instance, [16]) implies that the fixed point $u$ is bounded by $\left\|u_{0}\right\|_{\infty}$. Therefore, the solution can be extended for all times.

Moreover, when $u_{0} \geq 0$, the solution $u_{L}$ of the homogeneous equation (1.2) with initial datum $u_{0}$ is nonnegative. Thus, $u_{L}$ is a supersolution and 0 is a subsolution to (3.1). By the comparison principle we deduce that

$$
0 \leq u(x, t) \leq u_{L}(x, t)
$$

For the type of initial data we are interested in, much more can be said. In fact,

Theorem 3.1. Let $u_{0} \in L^{\infty}\left(\mathbb{R}^{N}\right), u_{0} \geq 0$ be such that $|x|^{\alpha} u_{0}(x) \rightarrow A>0$ as $|x| \rightarrow \infty$ with $\alpha, A>0$. Let $p>1$ and let $u$ be the solution to (1.1). Then,

(1) For every $T<\infty$, there exists a constant $C_{T}$ such that

$$
u(x, t) \leq C_{T}(1+|x|)^{-\alpha} \quad \text { for } t \leq T .
$$

(2) If $\alpha<N$, there exists a constant $C$ such that $u(x, t) \leq C t^{-\alpha / 2}$. If $\alpha=N$, there exists a constant $C$ such that $u(x, t) \leq C t^{-N / 2} \log t$.

(3) For every $t>0,|x|^{\alpha} u(x, t) \rightarrow A$ as $|x| \rightarrow \infty$ uniformly for $t$ in bounded sets.

Proof. (1) and (2) follow immediately from the estimate (3.2) above and the results for $u_{L}$ (Proposition 2.1 (1)).

In order to prove (3) we use the variations of constants formula

$$
u(x, t)=u_{L}(x, t)-\int_{0}^{t} S(t-s) u^{p}(x, s) d s .
$$

We already know that the first term on the right hand side has the correct limit uniformly for $t$ bounded (Proposition 2.1(2)). Thus, we have to prove that the second term goes to 0 faster than $|x|^{-\alpha}$.

We think of $u^{p}(x, s)$ as a nonnegative, bounded, initial condition that satisfies that for a constant $B$ that is independent of $s \in[0, T],|x|^{p \alpha} u^{p}(x, s) \leq B$. By 
Proposition 2.1 and Theorem 2.1 we know that

$$
|x|^{\alpha p} S(t-s) u^{p}(x, s) \leq C_{T} \quad \text { if } 0 \leq s \leq t \leq T,
$$

for a certain constant $C_{T}$. Thus,

$$
|x|^{\alpha} S(t-s) u^{p}(x, s)=|x|^{-\alpha(p-1)}|x|^{\alpha p} S(t-s) u^{p}(x, s) \leq C_{T}|x|^{-\alpha(p-1)}<\varepsilon
$$

if $|x|$ is large. Therefore,

$$
\left.|| x\right|^{\alpha} \int_{0}^{t} S(t-s) u^{p}(x, s) d x \mid<\varepsilon T \quad \text { if } \quad 0 \leq s \leq t \leq T \quad \text { and }|x| \text { is large. }
$$

\section{Asymptotic Behavior for the EQUation with ABSORPtion}

In this section we prove our main result, namely, that in the supercritical case, the solution to (1.1) with a bounded nonnegative initial datum $u_{0}$ satisfying (1.5) has the same asymptotic behavior as that of the homogeneous heat equation with diffusivity $\mathfrak{a}$.

Theorem 4.1. Let $u_{0} \geq 0, u_{0} \in L^{\infty}\left(\mathbb{R}^{N}\right)$ be such that $|x|^{\alpha} u_{0}(x) \rightarrow A>0$ as $|x| \rightarrow \infty$ with $0<\alpha \leq N$. Let $p>1+\frac{2}{\alpha}$. Let $u$ be the solution to (1.1) with $u(x, 0)=u_{0}(x)$.

Then, if $\alpha<N$,

$$
t^{\alpha / 2}\left\|u(x, t)-U_{\alpha, A}(x, t)\right\|_{L^{\infty}(|x| \leq K \sqrt{t})} \rightarrow 0 \quad \text { as } \quad t \rightarrow \infty \quad \forall K>0,
$$

where $U_{\alpha, A}$ is the solution to (1.7).

If $\alpha=N$,

$$
t^{N / 2}\left\|\frac{u(x, t)}{\log t}-C_{A, N} U_{a}(x, t)\right\|_{L^{\infty}(|x| \leq K \sqrt{t})} \rightarrow 0 \quad \text { as } t \rightarrow \infty \quad \forall K>0,
$$

where $U_{a}$ is the fundamental solution of the heat operator with diffusivity $\mathfrak{a}$ and $C_{A, N}$ is a constant that depends only on $A$ and $N$.

Proof. By Theorem 3.1 we know that $|x|^{\alpha} u\left(x, t_{0}\right) \rightarrow A$ as $|x| \rightarrow \infty$. Thus, by Theorem 2.1 we have that the solution $u_{L}$ to

$$
\begin{aligned}
& v_{t}=L v \quad \text { in } \mathbb{R}^{N} \times\left(t_{0}, \infty\right), \\
& v\left(x, t_{0}\right)=u\left(x, t_{0}\right)
\end{aligned}
$$

satisfies

$$
\begin{aligned}
& t^{\alpha / 2}\left\|u_{L}(x, t)-U_{\alpha, A}(x, t)\right\|_{L^{\infty}(|x| \leq K \sqrt{t})} \rightarrow 0 \quad \text { as } \quad t \rightarrow \infty \quad \forall K>0 \quad \text { if } \quad \alpha<N, \\
& t^{N / 2}\left\|\frac{u_{L}(x, t)}{\log t}-C_{A, N} U(x, t)\right\|_{L^{\infty}(|x| \leq K \sqrt{t})} \rightarrow 0 \quad \text { as } t \rightarrow \infty \quad \forall K>0 \quad \text { if } \quad \alpha=N .
\end{aligned}
$$

Thus, the theorem will be proved if we show that for every $\varepsilon>0$ there exists $t_{0}$ such that

$$
t^{\alpha / 2}\left\|u(x, t)-u_{L}(x, t)\right\|_{L^{\infty}\left(\mathbb{R}^{N}\right)} \leq \varepsilon \quad \text { for } t \geq 2 t_{0} .
$$

Here $u_{L}$ is the solution of (4.1).

In order to prove this result we need to estimate

$$
u(x, t)-u_{L}(x, t)=-\int_{t_{0}}^{t} S(t-s) u^{p}(x, s) d s .
$$

We begin with the case $\alpha<N$. 
Let us estimate the integrand $S(t-s) u^{p}(x, s)$. On the one hand, since by (3.2) and Theorem 2.1. $u(x, s) \leq C s^{-\alpha / 2}$, the maximum principle applied to the solutions of $v_{t}=L v$ renders the estimate

$$
0 \leq S(t-s) u^{p}(x, s) \leq C s^{-\alpha p / 2} .
$$

On the other hand,

$$
\begin{aligned}
S(t-s) u^{p}(x, s) & =e^{-(t-s)} u^{p}(x, s)+\int W(x-y, t-s) u^{p}(y, s) d y \\
& \leq e^{-(t-s)} s^{-\frac{\alpha}{2} p}+\|W(\cdot, t-s)\|_{q^{\prime}}\left\|u^{p}(\cdot, s)\right\|_{q} .
\end{aligned}
$$

As $p>1$ we can take $q=\frac{N}{\alpha}$. Then, $\|W(\cdot, t-s)\|_{q^{\prime}} \leq C(t-s)^{-\frac{N}{2 q}}=C(t-s)^{-\frac{\alpha}{2}}$. On the other hand, $\left\|u^{p}(x, s)\right\|_{L^{q}\left(\mathbb{R}^{N}\right)}=\|u(x, s)\|_{L^{p q}\left(\mathbb{R}^{N}\right)}^{p}$. Thus, since $q=\frac{N}{\alpha}>1$,

$$
\begin{aligned}
\left\|u^{p}(\cdot, s)\right\|_{q}^{\frac{1}{p}} & \leq\left\|e^{-s} u_{0}(\cdot)\right\|_{p q}+\left\|\int W(x-y, s) u_{0}(y) d y\right\|_{p q} \\
& \leq C e^{-s}+C\left[u_{0}\right]_{q, \infty}\|W(\cdot, s)\|_{r},
\end{aligned}
$$

with $1+\frac{1}{p q}=\frac{1}{r}+\frac{1}{q}$ and $\left[u_{0}\right]_{q, \infty}=\sup _{\lambda>0}\left(\lambda^{q}\left|\left\{\left|u_{0}\right|>\lambda\right\}\right|\right)^{1 / q}$ (see, for instance, [1], Theorem 1.4.24).

Since $u_{0}$ is bounded and $|x|^{\alpha} u_{0}(x) \rightarrow A$ as $|x| \rightarrow \infty$, we have $u_{0} \in L^{q, \infty}$ and

$$
\left\|u^{p}(\cdot, s)\right\|_{q}^{\frac{1}{p}} \leq C e^{-s}+C s^{-\frac{N}{2 r^{\prime}}}=C s^{-\frac{\alpha(p-1)}{2 p}},
$$

and hence,

Therefore,

$$
S(t-s) u^{p}(x, s) \leq C e^{-(t-s)} s^{-\frac{\alpha}{2} p}+C(t-s)^{-\frac{\alpha}{2}} s^{-\frac{\alpha}{2}(p-1)} \leq C(t-s)^{-\frac{\alpha}{2}} s^{-\frac{\alpha}{2}(p-1)} .
$$

Now we estimate the approximation error for $t \geq 2 t_{0}$. We need to separate the integral and use estimate (4.2) for $s \in(t / 2, t)$ and estimate (4.3) for $s \in\left(t_{0}, t / 2\right)$. We obtain, by using that $\frac{\alpha}{2}(p-1)-1 \geq 0$ (critical or supercritical cases),

$$
\begin{aligned}
\int_{t_{0}}^{t} S(t-s) u^{p}(x, s) d s & \leq C \int_{t_{0}}^{t / 2}(t-s)^{-\frac{\alpha}{2}} s^{-\frac{\alpha}{2}(p-1)} d s+C \int_{t / 2}^{t} s^{-\frac{\alpha}{2} p} d s \\
& \leq(t / 2)^{-\frac{\alpha}{2}} \int_{t_{0}}^{t / 2} s^{-\frac{\alpha}{2}(p-1)} d s+C \int_{t / 2}^{t} s^{-\frac{\alpha}{2} p} d s \\
& \leq C t^{-\frac{\alpha}{2}} t_{0}^{-\frac{\alpha}{2}(p-1)+1}+C t^{-\frac{\alpha}{2} p+1} .
\end{aligned}
$$

Thus, if $t_{0}$ is large enough and $t \geq 2 t_{0}$, we get, by using that we are in the supercritical case $p>1+2 / \alpha$,

$$
t^{\frac{\alpha}{2}} \int_{t_{0}}^{t} S(t-s) u^{p}(x, s) d s \leq C t_{0}^{-\frac{\alpha}{2}(p-1)+1}+C t^{-\frac{\alpha}{2}(p-1)+1}<\varepsilon .
$$

We remark that this is a sharp estimate that shows, in particular, that in the critical case, the absorption and the diffusion terms are of the same order.

Now we analyze the case $\alpha=N$.

We proceed as before. Estimate (4.2) is changed to

$$
0 \leq S(t-s) u^{p}(x, s) \leq C s^{-N p / 2} \log ^{p} s .
$$


For the equivalent to estimate (4.3) we have to proceed differently, since the general form of Young's inequality that we have used is not valid when $q=1$ as would now be the case.

We take instead $q=\frac{1}{1-\delta}$ with $\delta>0$ small to be chosen later (we could have proceeded in this way before, but we wouldn't have gotten the sharp estimate), and we use Young's inequality to get

$$
\left\|u^{p}(\cdot, s)\right\|_{L^{1}}=\|u(\cdot, s)\|_{L^{p}}^{p} \leq C e^{-p s}+\|W(\cdot, s)\|_{L^{r}}^{p}\left\|u_{0}\right\|_{L^{q}}^{p},
$$

with $1+\frac{1}{p}=\frac{1}{r}+\frac{1}{q}$, so that $\frac{1}{r^{\prime}}=1-\delta-\frac{1}{p}$ and $\frac{N p}{2 r^{\prime}}=\frac{N}{2}(p-1-\delta p)$. Thus,

$$
\left\|u^{p}(\cdot, s)\right\|_{L^{1}} \leq C e^{-p s}+C_{\mu} s^{-\frac{N}{2}(p-1)+\mu} \leq C_{\mu} s^{-\frac{N}{2}(p-1)+\mu},
$$

with $\mu=\frac{N}{2} \delta p$ as small as needed.

Therefore,

$$
\begin{aligned}
S(t-s) u^{p}(x, s) & \leq e^{-(t-s)} s^{-\frac{N}{2} p} \log ^{p} s+\|W(\cdot, t-s)\|_{\infty}\left\|u^{p}(\cdot, s)\right\|_{1} \\
& \leq C e^{-(t-s)} s^{-\frac{N}{2} p} \log ^{p} s+C_{\mu}(t-s)^{-\frac{N}{2}} s^{-\frac{N}{2}(p-1)+\mu} \\
& \leq C_{\mu}(t-s)^{-\frac{N}{2}} s^{-\frac{N}{2}(p-1)+\mu} .
\end{aligned}
$$

As before, we use (4.4) and (4.5) to estimate the error. We have, by taking $0<\mu<\frac{N}{2}(p-1)-1$,

$$
\begin{aligned}
\left\|u(x, t)-u_{L}(x, t)\right\|_{L^{\infty}\left(\mathbb{R}^{N}\right) \leq} & \left\|\int_{0}^{t} S(t-s) u^{p}(x, s) d s\right\|_{L^{\infty}\left(\mathbb{R}^{N}\right)} \\
\leq & C_{\mu} \int_{t_{0}}^{t / 2}(t-s)^{-\frac{N}{2}} s^{-\frac{N}{2}(p-1)+\mu} d s \\
& +C \int_{t / 2}^{t} s^{-\frac{N}{2} p} \log ^{p} s d s \\
\leq & C_{\mu}(t / 2)^{-\frac{N}{2}} t_{0}^{-\frac{N}{2}(p-1)+1+\mu}+C t^{-\frac{N}{2} p+1} \log ^{p} t,
\end{aligned}
$$

so that, since $p>1+2 / N$, if $t_{0}$ is large enough and $t \geq 2 t_{0}$, we get

$$
t^{N / 2}\left\|u(x, t)-u_{L}(x, t)\right\|_{L^{\infty}\left(\mathbb{R}^{N}\right)} \leq C_{\mu} t_{0}^{-\frac{N}{2}(p-1)+1+\mu}+C t^{-\frac{N}{2}(p-1)+1} \log ^{p} t<\varepsilon .
$$

So, the theorem is proved.

\section{REFERENCES}

[1] P. Bates, A. Chmaj, An integrodifferential model for phase transitions: Stationary solutions in higher dimensions, J. Statistical Phys. 95, 1999, 1119-1139. MR1712445 $(2000 \mathrm{j}: 82020)$

[2] P. Bates, A. Chmaj, A discrete convolution model for phase transitions, Arch. Rat. Mech. Anal. 150, 1999, 281-305. MR1741258 (2001c:82026)

[3] P. Bates, P. Fife, X. Ren, X. Wang, Travelling waves in a convolution model for phase transitions, Arch. Rat. Mech. Anal. 138, 1997, 105-136. MR1463804(98f:45004)

[4] C. Carrillo, P. Fife, Spatial effects in discrete generation population models, J. Math. Biol. 50(2), 2005, 161-188. MR2120547 (2005k:92048)

[5] M. Chaves, E. Chasseigne, J. D. Rossi, Asymptotic behavior for nonlocal diffusion equations, J. Math. Pures Appl. (9) 86(3), 2006, 271-291. MR2257732 (2007e:35279)

[6] C. Cortazar, M. Elgueta, F. Quiros, N. Wolanski, Large time behavior of the solution to the Dirichlet problem for a nonlocal diffusion equation in an exterior domain, in preparation. 
[7] C. Cortazar, M. Elgueta, J. D. Rossi, Nonlocal diffusion problems that approximate the heat equation with Dirichlet boundary conditions, Israel Journal of Mathematics 170(1), 2009, 53-60. MR 2506317 (2010e:35197)

[8] C. Cortazar, M. Elgueta, J. D. Rossi, N. Wolanski, How to approximate the heat equation with Neumann boundary conditions by nonlocal diffusion problems, Arch. Rat. Mech. Anal. 187(1), 2008, 137-156. MR2358337(2008k:35261)

[9] P. Fife, Some nonclassical trends in parabolic and parabolic-like evolutions, Trends in Nonlinear Analysis, 153-191, Springer, Berlin, 2003. MR 1999098(2004h:35100)

[10] G. Gilboa, S. Osher, Nonlocal operators with application to image processing, Multiscale Model. Simul. 7(3), 2008, 1005-1028. MR2480109(2010b:94006)

[11] L. Grafakos, Classical Fourier analysis. Second edition. Graduate Texts in Mathematics, 249. Springer, New York, 2008. MR2445437

[12] L. Herraiz, Asymptotic behaviour of solutions of some semilinear parabolic problems, Ann. Inst. Henri Poincaré 16(1), 1999, 49-105. MR1668560 (2000a:35106)

[13] L. I. Ignat, J. D. Rossi, Refined asymptotic expansions for nonlocal diffusion equations, J. Evolution Equations. 8, 2008, 617-629. MR2460931 (2009j:45001)

[14] S. Kamin, L. A. Peletier, Large time behavior of solutions of the heat equation with absorption, Anal. Scuola. Norm. Sup. Pisa Serie 4, 12, 1985, 393-408. MR837255 (87h:35140)

[15] S. Kamin, M. Ughi, On the behaviour as $t \rightarrow \infty$ of the solutions of the Cauchy problem for certain nonlinear parabolic equations, J. Math. Anal. Appl. 128, 1997, 456-469. MR917378(89m:35029)

[16] C. Lederman, N. Wolanski, Singular perturbation in a nonlocal diffusion model, Communications in PDE 31(2), 2006, 195-241. MR2209752 (2007e:35166)

[17] A. Pazoto, J. D. Rossi, Asymptotic behaviour for a semilinear nonlocal equation. Asymptotic Analysis 52(1-2), 2007, 143-155. MR2337030(2008h:35023)

[18] J. Terra, N. Wolanski, Large time behavior for a nonlocal diffusion equation with absorption and bounded initial data, submitted.

[19] L. Zhang, Existence, uniqueness and exponential stability of traveling wave solutions of some integral differential equations arising from neuronal networks, J. Differential Equations 197(1), 2004, 162-196. MR2030153 (2004m:45010)

Departamento de Matemática, Facultad de Ciencias Exactas y Naturales, Universidad de Buenos Aires, (1428) Buenos Aires, Argentina

E-mail address: jterra@dm.uba.ar

Departamento de Matemática, Facultad de Ciencias Exactas y Naturales, UniversiDAd de Buenos Aires, (1428) Buenos Aires, Argentina

E-mail address: wolanski@dm.uba.ar 